\title{
Optical Limiting and Stabilization Properties of a Liquid Dye on 1064 nm Nanosecond Laser Pulses
}

\author{
Liuheng Wang ${ }^{1,2}$, Rongzong Peng ${ }^{1,2}$, Yuxia Zhao ${ }^{1}$, Feipeng $\mathrm{Wu}^{1}$ \\ ${ }^{1}$ Technical Institute of Physics and Chemistry, Chinese Academy of Sciences, Beijing, P. R. China \\ ${ }^{2}$ Graduate University of Chinese Academy of Sciences, Beijing, P. R. China \\ Email: fpwu@mail.ipc.ac.cn
}

Received 2013

\begin{abstract}
A novel liquid dye 2-((2E,5E)-2,5-bis(4-(methyl(2,5, 8,11-tetraoxatridecan-13-yl)amino)benzylidene)cyclopentylidene) malononitrile (TO-BDCM) was synthesized by incorporating two oligo(ethenyl glycol) groups into the backbone of a prototype malononitrile derivative (BDCM) for the purpose of increasing its solubility in organic solvents. The nonlinear absorption properties of this liquid dye on $1064 \mathrm{~nm}$ ns pulsed laser were investigated in DMF with remarkably high concentration up to $\sim 1 \mathrm{M}$, showing a superior large nonlinear absorption coefficient of $55.6 \mathrm{~cm}^{3} / \mathrm{GW}^{2}$. Fairly good optical limiting and stabilization effects were achieved. Meanwhile, the liquid dye exhibited equivalent stability under laser irradiation compared to its prototype dye BDCM.
\end{abstract}

Keywords: Liquid Dye; Multiphoton Absorption; Optical Limiting; Optical Stabilization

\section{Introduction}

Optical limiting and stabilization techniques become more and more important as the fast development of ultra short pulsed lasers. Using these techniques can protect human eyes and optical sensors from damages induced by intense laser pulses, or stabilize the temporal fluctuation of laser pulses to keep the peak energy of all pulses at a certain level $[1,2]$. Materials based on multiphoton absorption are most widely used to fabricate optical limiter or stabilizer because of their fast response of the ultra short pulses, as well as the remarkably high linear transmission at low energy, compared with other materials, such as reverse saturable absorption or induced scattering materials $[3,4]$.

In order to improve the optical limiting and stabilization properties of multiphoton absorption (MPA) materials, researchers have reported a number of methods to increase the multiphoton absorption cross section of MPA materials, such as elongating the conjugation length of the molecule, or using a dendritic structure [5]. However, in most of the cases, the solubility of such materials in organic solvents is a big problem. Except to increase the multiphoton absorption cross section of materials, to increase the concentration of a given materials is also an effective strategy for enhancing its optical limiting and stabilization performance [6].

In our previous work, we have reported a malononitrile derivative (BDCM) with relatively large two-photon absorption cross section [7], and can be potential choice as good optical limiter and stabilizer. But the maximum concentration of the dye in organic solvents limits to $\sim 10^{-2} \mathrm{M}$, which may restrict its optical limiting properties in some extent. In order to increase the solubility, In this work, oligo(ethenyl glycol) groups were introduced to the backbone of BDCM and 2-((2E,5E)-2,5-bis (4(methyl(2,5,8,11-tetraoxatridecan-13yl)amino)benzylidene) cyclopentylidene) malononitrile (TO-BDCM) was obtained. It is a dark-blue with highly viscous oil at room temperature. By mixing with one or two parts (mess ratio) of DMF, a uniform optical solution can be obtained. The solubility of TO-BDCM is remarkably increased to a magnitude larger than that of BDCM. The high concentration of effective dyes in the solution should significantly improve the final optical limiting and stabilization performance of TO-BDCM.

\section{Results and Discussion}

\subsection{Synthesis}

The chemical structure and synthetic procedure of TOBDCM is shown in Figure 1. Two oligo (ethenyl glycol) groups were incorporated into BDCM to decrease the glass transition temperature and improve its solubility in organic solvents. The target liquid dye TO-BDCM was obtained through Knoevenagel condensation between the benzaldehyde 1 [8] and 2-cyclopentylidenemalononitrile. It was a dark-blue and highly viscous oil at room temperature. 


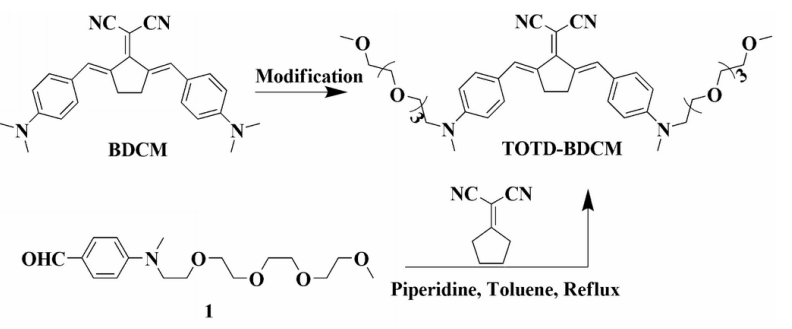

Figure 1. Chemical structure and synthetic route of the liquid chromophore TO-BDCM.

2-((2E,5E)-2,5-bis(4-(methyl(2,5,8,11-tetraoxatridecan-1 3-yl)amino)benzylidene)cyclopentylidene)malononitrile( TO-BDCM). Yield $42 \%$. ${ }^{1} \mathrm{H}$ NMR $\left(\mathrm{CDCl}_{3}, 400 \mathrm{MHz}\right)$ $\delta 8.04(\mathrm{~s}, 2 \mathrm{H}), 7.42(\mathrm{~d}, 4 \mathrm{H}, \mathrm{J}=8.9 \mathrm{~Hz}), 6.75(\mathrm{~d}, 4 \mathrm{H}$, $\mathrm{J}=8.9 \mathrm{~Hz}), 3.69-3.60$ (m, $28 \mathrm{H}), 3.55-3.53(\mathrm{~m}, 4 \mathrm{H}), 3.37$ (s, $6 \mathrm{H}), 3.08$ (s, $6 \mathrm{H}), 2.91$ (s, $4 \mathrm{H})$.

\subsection{Optical Properties}

The linear attenuation of pure TO-BDCM and its DMF solution was as shown as in Figure 2. From Figure 2 one can see that the transmission of the pure TO-BDCM was much lower than its DMF solution. This is because the refractive index distribution in the viscous TO-BDCM is not homogeneous. So the TO-BDCM can not be directly applied in optical limiting and stabilization as its original form. Figure 2 shows that the linear attenuation of the DMF solution is less than 1\% at $1064 \mathrm{~nm}$ (after subtracting the effect of the cuvette). As a result, diluted DMF solution is the better sample medium for optical limiting and stabilization application, compared with pure TO-BDCM. The linear attenuation was carried out on a Virian Cary 5000 UV-vis-NIR spectrophotometer. The DMF solution was prepared by mixing DMF and TO-BDCM in volume ratio of $\sim 1 / 1$. The solution concentration was about $\sim 0.75 \mathrm{M}$. Both the pure TO-BDCM and the DMF solution were filled in 1-cm length cuvette.

The UV-Vis and fluorescence spectrum of TO-BDCM and BDCM were shown in Figure 2. From the spectrum one can see that the maximum absorption of TO-BDCM was located at $577 \mathrm{~nm}$. This indicates that possible twophoton absorption may occur around $1064 \mathrm{~nm}$. Both the $\mathrm{UV}-\mathrm{Vis}$ and fluorescence spectrum of TO-BDCM are similar to BDCM, so the incorporation of oligo(ethenyl glycol) groups to the backbone has little influence on both ground and excited state of BDCM. The UV-Vis spectrum was measured on a Hitachi U3900 spectrophotometer by using $1 \times 10^{-5} \mathrm{M}$ DMF solution. The fluorescence spectrum was investigated on a Hitachi F4500 FL spectrophotometer. For the fluorescence spectrum measurement, the concentration of the DMF solution was $\sim 10^{-4} \mathrm{M}$, besides, the exciting and emission slit of the spectrophotometer were set to the largest. This is because the Fluorescence quantum yield of these two compounds are very low.

\subsection{Photostability}

The photobleaching of TO-BDCM and BDCM is shown in Figure 3. $\ln \left(\mathrm{A} / \mathrm{A}_{0}\right)$ is plotted as a function of irradiation time, where $A_{0}$ and $A$ were the maximum absorption before and after every $10 \mathrm{~min}$ of irradiation. The linear fitting of the plot yields the first order rate constant of 6.2 $\times 10^{-4} / \mathrm{min}$ and $6.6 \times 10^{-4} / \mathrm{min}$ for BDCM and TOBDCM, respectively. TO-BDCM and BDCM exhibit equivalent photochemical stability. The photobleaching was carried out by irradiating the DMF solution filled in $1-\mathrm{cm}$ cuvette with a $532 \mathrm{~nm}$ Semiconductor solid-state laser (MGL-532 nm-III-30 mW, Changchun New Industries Optoelectronics Tech. Co., Ltd). The solutions are prepared with the same absorption at $532 \mathrm{~nm}$. The UVVis spectrum was measured every $10 \mathrm{~min}$ of irradiation

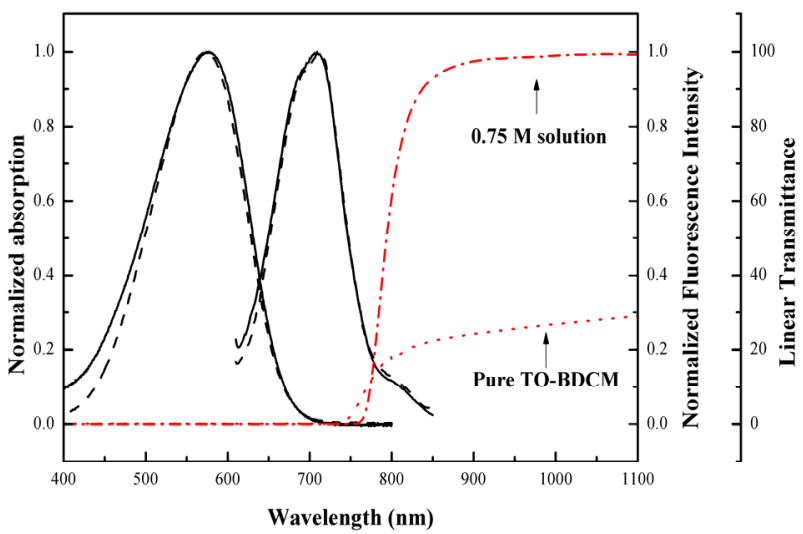

Figure 2. UV-Vis and fluorescence spectrum for diluted TO-BDCM (solid lines) and BDCM (dashed lines) solutions; Linear transmission curve of pure TO-BDCM filled in 1-mm cuvette (dot line) and a slightly diluted solution of $0.75 \mathrm{M}$ in DMF (dash-dot line).

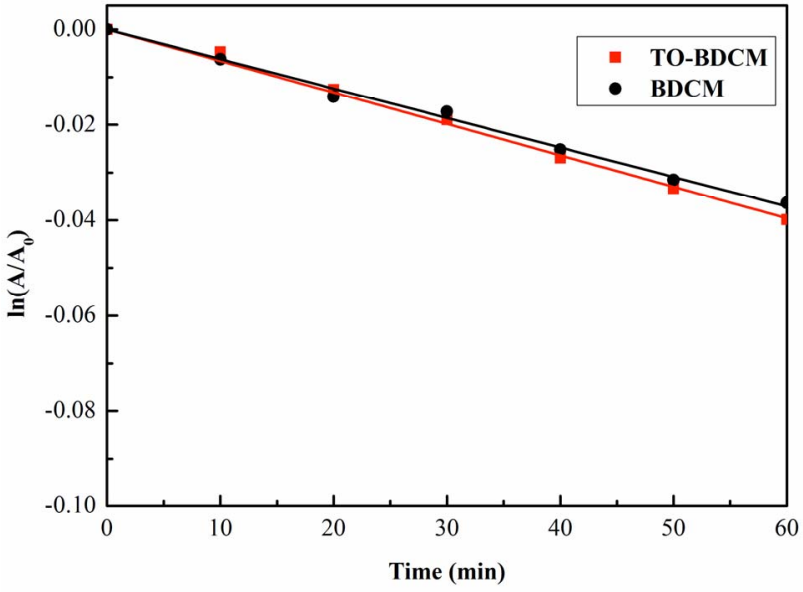

Figure 3. Plot of $\ln (\mathrm{A} / \mathrm{A} 0)$ vs irradiation time, the slope of the linear fitting curve is the first order photobleaching rate constant. 
on Hitachi-U3900 UV-Vis spectrophotometer. The concentrated solution of $\sim 0.75 \mathrm{M}$ was also directly irradiated by $1064 \mathrm{~nm}$ pulsed laser (Quantum Ray). The pulse duration, repetition rate and beam radius were $8 \mathrm{~ns}, 10 \mathrm{~Hz}$, and $45 \mathrm{~mm}$, respectively. The beam diameter was smaller than the width of the cuvette so that the entire of the beam can pass through the sample solution. In this condition, the pulse intensity was about $0.2 \mathrm{GW} / \mathrm{cm}^{2}$. After irradiation of $2 \mathrm{~h}$, the solution was diluted to $\sim 10^{-5} \mathrm{M}$ and then the UV-Vis spectrum was measured on Hitachi U-3900 spectrophotometer. we found that there was little change between the UV-Vis spectrum before and after irradiation. The liquid chromatography also found no new substance generated. This result may also indicate a good photochemical stability of TO-BDCM.

\subsection{Optical Limiting and Stabilization}

For all solution samples applied in optical limiting and stabilization, the multi-photon absorption (MPA) properties are proportional to the effective concentration of the solutions. The dye BDCM has been reported as an effective optical limiter on $1064 \mathrm{~nm}$ fs pulsed laser [8]. It has large two-photon absorption (TPA) cross section as hundreds of GM. But the effective concentration of this dye is limited to $\sim 10^{-2} \mathrm{M}$. After modifying the molecular, the effective concentration of TO-BDCM can reach up to $\sim 1$ M.

In nonlinear absorption experiment, a $1064 \mathrm{~nm} \mathrm{Nd}$ YAG pulsed laser (Quanta-Ray, Spectra Physics) was used as the excitation beam with pulse duration $8 \mathrm{~ns}$ and repetition rate $10 \mathrm{~Hz}$. The laser beam was focused by a lens $(f=500 \mathrm{~mm})$ and then passed through a cuvette filled with sample solutions. The cuvette was placed between the focus and the lens in the experiment, so that the laser beam in the sample solutions can approximately be supposed as parallel in this case. Meanwhile, this arrangement also can avoid the damage of the cuvette by the focused beam. In the case of ns pulsed laser, it is common to observe stronger nonlinear absorption compared to that in fs case because of the TPA induced excited state absorption. In such a case, a three-photon absorption model was more effective to be used to evaluate the experimental data compared to a two-photon absorption model. The plot of the output intensity vs input intensity can be fitted by $(1)[9,10]$

$$
I=\frac{I_{0}}{\sqrt{1+2 \gamma L I_{0}^{2}}}
$$

where $I$ was the output intensity, $I_{0}$ was the iput intensity, $\gamma$ was the effective three-photon absorption coefficient, and $L$ was the path length of the sample solutions.

Figure 4 (a) shows the optical limiting behavior of the $\sim 0.75 \mathrm{M}$ TO-BDCM solution filled in a $1-\mathrm{cm}$ cuvette.
The output intensity was plotted as a function of input intensity. One can see that the transmitted/input intensity characteristic curve starts to deviate from the linear transmission at about $0.05-0.06 \mathrm{GW} / \mathrm{cm}^{2}$, and becomes flattened when the input intensity is higher than about $0.10 \mathrm{GW} / \mathrm{cm}^{2}$. Specifically, in the experimental condition, the input intensity increased from 0.01 to $0.81 \mathrm{GW} / \mathrm{cm}^{2}$ (81 times increase), while the output intensity only increased from 0.01 to $0.10 \mathrm{GW} / \mathrm{cm}^{2}$ (10 times increase). The solid line is the theoretical curve predicted by (1), and the nonlinear fitting gives $\gamma$ as large as $55.6 \mathrm{~cm}^{3} / \mathrm{GW}^{2}$.

Figure 4(b) shows the nonlinear transmission as a function of input intensity. The solid line is the best fitting curve using the three-photon absorption theory with the $\gamma$ value obtained above. In the experimental condition, the nonlinear transmission is as low as $12.6 \%$ at the highest input intensity.

For most pulsed laser-based application, the intensity stability is very important. In other words, the fluctuation of the intensity among different pulses is harmful. MPA materials are one of the best approaches to stabilize the pulse energy. From Figure 4(a) one can see that in the experimental condition, when the intensity of the input pulses is higher than $0.10 \mathrm{GW} / \mathrm{cm} 2$, the optical limiting curve becomes flat, and the output intensity tends to a fixed value of $\sim 0.10 \mathrm{GW} / \mathrm{cm} 2$. So if the intensity of the input pulses varies between $0.10 \mathrm{GW} / \mathrm{cm} 2$ and any value larger than $0.10 \mathrm{GW} / \mathrm{cm} 2$, the intensity of transmitted pulses fluctuates around $0.10 \mathrm{GW} / \mathrm{cm} 2$ within a small variation range. This is a simple example for the prin ciple of optical stabilization based on MPA.

Figure 5 shows the optical stabilization of the $0.75 \mathrm{M}$ TO-BDCM solution filled in a $2-\mathrm{mm}$ cuvette. The transmitted and input beam power were measured by two
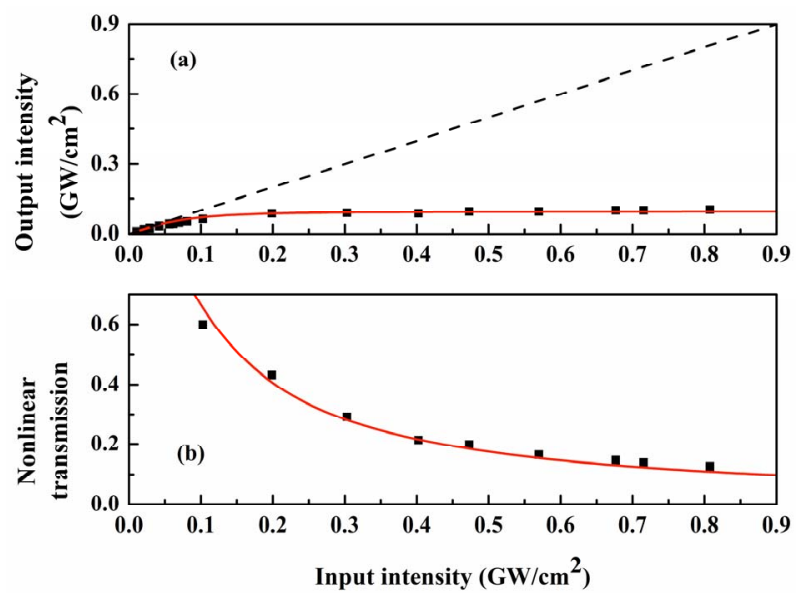

Figure 4. (a) Output intensity as a function of input intensity, dashed and solid line represent the theoretical linear and nonlinear absorption curve, respectively, (b) Nonlinear transmission as a function of input intensity, solid line represents theoretical nonlinear transmission curve. 


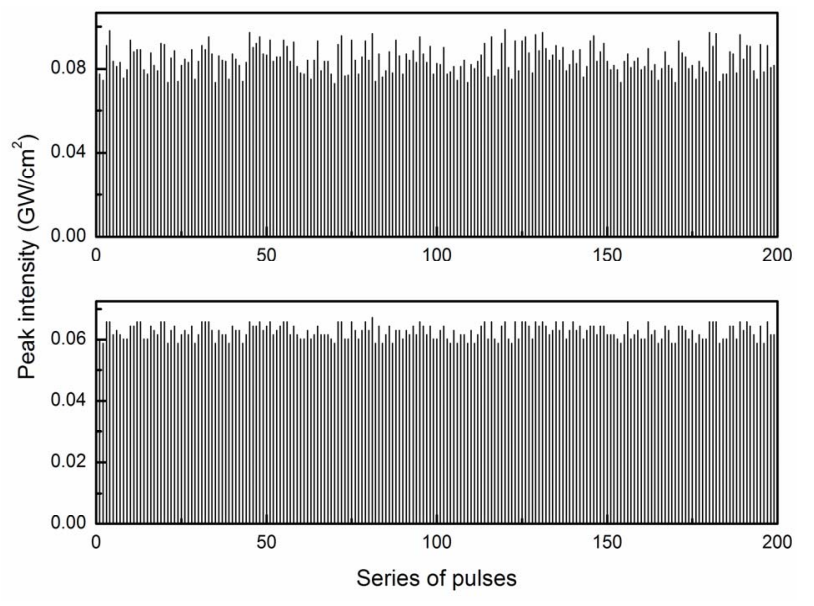

Figure 5. Fluctuation of input (upper trace) and transmitted intensity (lower trace).

thermopile energy detectors (OPHIR, LASERSTAR DUAL CHANNEL METER ASSY, RoHS, 50A-PF-DIF-18) simultaneously.The upper and the lower trace is the intensity fluctuation of input and transmitted pulses, respectively. It shows that when the input intensity changes from $0.073 \mathrm{GW} / \mathrm{cm}^{2}$ to $0.099 \mathrm{GW} / \mathrm{cm}^{2}$, with the fluctuation of $26.3 \%$, the output intensity changes from 0.059 $\mathrm{GW} / \mathrm{cm}^{2}$ to $0.067 \mathrm{GW} / \mathrm{cm}^{2}$, with a reduced fluctuation of $11.9 \%$. There is a 2 times reduction of intensity fluctuation after passing through a $2-\mathrm{mm}$ thick TO-BDCM solution. So the DMF solution of TO-BDCM is a good optical stabilizer.

\section{Conclusions}

In conclusion, we have synthesized a new liquid compound TO-BDCM by incorporating oligo(ethenyl glycol) groups into the backbone of a prototype chromophore BDCM. TO-BDCM has remarkable solubility in organic solvent. Its effective concentration can reach up to $\sim 1 \mathrm{M}$ in DMF, which results in remarkable nonlinear absorbability. The solution of this liquid dye with high effective concentration shows fairly good optical limiting and stabilization behavior on $1064 \mathrm{~nm}$ ns pulsed laser. Meanwhile, the liquid dye shows equivalent photochemical stability under irradiation compared to its prototype compound BDCM.

\section{Acknowledgements}

This research was supported by NSAF projects (10776033, U1230123).

\section{REFERENCES}

[1] G. S. He, L.-S. Tan, Q. D. Zheng and P. N. Prasad, "Multiphoton Absorbing Materials: Molecular Designs, Characterizations and Applications," Chemical Reviews, Vol. 108, No. 4, 2008, pp. 1245-1330. doi:10.1021/cr050054x

[2] L. W. Tutt and T. F. Boggess, "A Review of Optical Limiting Mechanisms and Devices Using Organics, Fullerenes, Semiconductors and Other Materials," Progress in Quantum Electronics, Vol. 17, No. 4, 1993, pp. 299-338. doi:10.1016/0079-6727(93)90004-S

[3] C. R. Giuliano and L. D. Hess, "Nonlinear Absorption of Light: Optical Saturation of Electronic Transition in Organic Molecules with High Intensity Laser Radiation," IEEE Journal of Quantum Electronics, Vol. 3, No. 8, 1967, pp. 358-367. doi:10.1109/JQE.1967.1074603

[4] G. S. He, C. Weder, P. Smith and P. N. Prasad, "Optical Limiting and Stabilization Based on A Novel Polymer Compound," IEEE Journal of Quantum Electronics, Vol. 34, No. 12, 1998, pp. 2279-2285. doi:10.1109/3.736090

[5] S.-J. Chung, T.-C. Lin, K.-S. Kim, G. S. He, J. Swiatkiewicz, P. N. Prasad, G. A. Baker and F. V. Bright, "Two-Photon Absorption and Excited-State Energy-Transfer Properties of A New Multibranched Molecule," Chemistry of Materials, Vol. 13, No. 11, pp. 4071-4076, 2001. doi:10.1021/cm010151g

[6] G. S. He, Q. D. Zheng, C. G. Lu and P. N. Prasad, "Two-and Three-Photon Absorption Based Optical Limiting and Stabilization Using A Liquid Dye," IEEE Journal of Quantum Electronics, Vol. 41, No. 7, 2005, pp. 1037-1043. doi:10.1109/JQE.2005.848913

[7] J. X. Guan, Y. X. Zhao, F. P. Wu, X. Y. Fang and J. F. Zhao, "Solvent Effect on Optical Limiting and Anti-Damage Properties of Dicyanomethylene Derivatives at 1064 nm," SPIE Proceedings, Laser-Induced Damage in Optical Materials, Vol. 7132, 2008, pp. 7132131-7132138.doi:10.1117/12.804410

[8] Y. X. Zhao, W. J. Wang, F. P. Wu, Y. Zhou, N. Y. Huang, Y. Gu, Q. L. Zou and W. Yang, "Polyethylene Glycol-Functionalized Benzylidene Cyclopentanone Dyes for Two-Photon Excited Photodynamic Therapy," Organic and Biomolecular. Chemistry, Vol. 9, No. 11, 2011, pp. 4168-4175. doi:10.1039/c0ob01278e

[9] G. S. He, J. D. Bhawalkar, P. N. Prasad and B. A. Reinhardt, "Three-Photon-Absorption-Induced Fluorescence and Optical Limiting Effects in An Organic Compound," Optics. Letters, Vol. 20, No. 14, 1995, pp. 1524-1526. doi:10.1364/OL.20.001524

[10] J. H. Liu, and Y. X. Wang, "Three-Photon-Absorption Induced Optical Stabilization Effects in A Bifluorenylidene Derivative," Optics Express, Vol. 20, No. 13, 2012, pp. 14596-14603. doi:10.1364/OE.20.014596 